\title{
Fractal and Morphological Characteristics of Single Marble Particle Crushing in Uniaxial Compression Tests
}

\author{
Yidong Wang, ${ }^{1}$ Wenjiao Dan, ${ }^{2}$ Yongfu $\mathrm{Xu},{ }^{1}$ and Yue $\mathrm{Xi}^{1}$ \\ ${ }^{1}$ Department of Civil Engineering, Shanghai Jiao Tong University, Shanghai 200240, China \\ ${ }^{2}$ Department of Engineering Mechanics, Shanghai Jiao Tong University, Shanghai 200240, China \\ Correspondence should be addressed to Yongfu Xu; yongfuxu@sjtu.edu.cn
}

Received 12 September 2015; Revised 24 November 2015; Accepted 25 November 2015

Academic Editor: Pavel Lejcek

Copyright (c) 2015 Yidong Wang et al. This is an open access article distributed under the Creative Commons Attribution License, which permits unrestricted use, distribution, and reproduction in any medium, provided the original work is properly cited.

\begin{abstract}
Crushing of rock particles is a phenomenon commonly encountered in geotechnical engineering practice. It is however difficult to study the crushing of rock particles using classical theory because the physical structure of the particles is complex and irregular. This paper aims at evaluating fractal and morphological characteristics of single rock particle. A large number of particle crushing tests are conducted on single rock particle. The force-displacement curves and the particle size distributions (PSD) of crushed particles are analysed based on particle crushing tests. Particle shape plays an important role in both the micro- and macroscale responses of a granular assembly. The PSD of an assortment of rocks are analysed by fractal methods, and the fractal dimension is obtained. A theoretical formula for particle crushing strength is derived, utilising the fractal model, and a simple method is proposed for predicting the probability of particle survival based on the Weibull statistics. Based on a few physical assumptions, simple equations are derived for determining particle crushing energy. The results of applying these equations are tested against the actual experimental data and prove to be very consistent. Fractal theory is therefore applicable for analysis of particle crushing.
\end{abstract}

\section{Introduction}

Granular materials are widely used in rock fill dams, highways, railways, and dykes, due to their engineering properties such as high hydraulic permeability, high density, high shear strength, and low settlement. Coarse particles are susceptible to particle crushing at a high compressive strength, which directly modifies their structure, influencing dilatancy, friction angle, strength, and permeability [1]. The strength of granular material decreases during particle crushing as its compression increases, which may eventually lead to significant deformations and ultimately to structural instability [2]. Determining the exact mechanics of particle crushing, or particle breakage of granular material, is one of the most intractable problems in the geosciences. This topic is of interest to many subfields of research including powder technology, minerals and mining engineering, geology, geophysics, and geomechanics [3].

The problems associated with particle crushing in geomechanics began to attract attention in the early 1960s and from then on gradually developed into a topic of significant study. Research on particle crushing has since been carried out via four approaches. Firstly, researchers have experimented with various artificial materials. Takei et al. [4] carried out single particle compressive strength testing and one-dimensional compression testing with plaster and talc sticks, glass beads, and quartz, discussing the fragmentation mechanism of these four different materials in detail. Secondly, studies have attempted to mathematically describe the crushing characteristics of particles, which is of particular interest to the field of geomechanics. They linked the crushing behaviour of particles and their mechanical response through the use of behavioural constitutive models. Existing constitutive models are based on simple curve-fitting parameters, which are determined in isolation by discrete stress-strain tests. Nakata et al. [5] conducted single particle crushing tests on three kinds of particles and analysed the results using particle survival probability curves. Matsui et al. [6] investigated methods for estimating the ratio of net work input to crushing and specific surface area produced based on load conditions 
and material properties. Rozenblat et al. [7] expressed particle strength distributions based on an extended logistic function of the crushing force of a number of individual particles. Thirdly, the mechanical properties of crushed particles on the macroscopic level were determined. The macroscopic mechanical properties of particle crushing have been studied through direct shear tests, ring shear tests, uniaxial compression tests, and triaxial shear tests, which mainly characterised the strength and the deformation [8] characteristics of the particles. Lastly, mesoexperiments on particle crushing were carried out through the use of some advanced instruments and methods such as electron microscopy and X-ray [9] in order to obtain detailed structural information.

The majority of analyses focused on experimentally determining the physical characteristics of single particle crushing. Russell et al. [10-12] analysed breakage behaviour of characteristic elastic-plastic granules using compression tests. The study describes the influences of granule size, moisture content, and loading intensity on the energy absorption and recovery at stressing. Mader-Arndt et al. [13] investigated the particle contacts by means of atomic force microscopy (AFM), nanoindentation, and shear tests. Ribas et al. [14] and Portnikov et al. [15] designed compression testers to accurately measure the force-displacement curves, the distribution of strengths, and the fracture energies of single particles.

Mandelbrot [16] has however observed that several natural phenomena can be accurately described by fractal theory. The fractal dimension, $D$, is a fraction with a value between 0 and 3.0. Fractals are self-similar objects, allowing the fractal fragment size distribution at any scale to be predicted [17]. If the shape of some rock fragments is fractal, then their fractal dimension may hence be estimated from their size distribution [18]. Prior studies have generally been aimed towards characterising the particle size distribution (PSD). Many statistical methods have been proposed for describing the PSD of comminuted materials, and the most significant of such PSD functions, including normal, log-normal, GatesGaudin-Schumann, and Rosin-Rammler distribution functions, have been reviewed in detail by Allen [19]. The use of fractal size distribution analysis is another approach for linearizing the size distribution curve [20-22]. The concept of utilising probability for studying particle crushing was introduced by McDowell et al. [23], who described a method whereby the compression behaviour of particles could be expressed using a probabilistic approach based around the mathematics of fractals. Xu et al. [24] expressed a significant "size effect" in ice failure strength and used the fractal model for studying ice particle fragmentation by employing modified Weibull [25] statistics. Combining traditional experimental methods with insights from fractal theory is hence an effective method for determining the morphology and mechanical characteristics of particle crushing.

This paper aims at exploring fractal crushing characteristics of single particle. Crushing tests on various sized particles are carried out to evaluate the crushing characteristics of each individual particle. Particle shape plays an important role in both the micro- and macroscales responses of a granular assembly [26]. The relationships between failure modes and particle shapes are analysed, and the fractal model is employed to describe the particle crushing behaviour. The fractal dimension $D$ of the particle size distribution is determined and found to be equal to 2.48 for marble particles. The crushing strength is then connected to the particle size combining with the fractal fragmentation of marble particles. The Weibull statistics was modified to estimate the probability of fracture for marble particles, and the Weibull modulus was determined using the fractal dimension of particle fragmentation. The formula of the size effect on the crushing energy is also analysed using fractal model for particle fragmentation.

\section{Fractal Model for Particle Crushing}

A fractal is a shape consisting of parts similar to the whole in some manner [16]. A certain value is usually used to express the similarity between the parts and the entirety, which is termed the fractal dimension. The fractal dimension attempts to objectively represent how densely a fractal occupies the metric space in which it lies. Fractal dimensions are important because they can be defined in relation to real-world material behaviour and can hence be measured experimentally.

A fractal particularly suited to the analysis of particle crushing is that defined as a box-counting measure $N(d)$, the number of particles having diameters greater than or equal to $d$, which displays scale invariance with a noninteger exponent [27]:

$$
N(r) \propto d^{-D}
$$

In (1), the fractal dimension $D$ can be estimated from the slope of a straight line in the log-log plot. The number of particles $N(d)$ is very difficult to count accurately, but the mass of the particles may easily be measured. A simple approach to calculating the fractal dimension of particle crushing is the following algorithm [18]:

$$
\mathrm{d} M \propto d^{3} \mathrm{~d} N \propto d^{2-D},
$$

where $M$ is the total mass of the particles.

If the particles are screened after crushing with a sieve of aperture $d$, the total mass of the particles under the sieve of aperture $d_{i}$ is $M\left(d_{i}\right)$ and the total mass of all the particles is $M_{T}$.

The particle density is a constant value, so the mass of the particles of diameter less than $d_{i}$ can be expressed as

$$
M\left(d_{i}\right)=\int \kappa \rho d^{3} \mathrm{~d} N(r) \propto d^{3-D},
$$

where $\kappa$ is a shape factor. Equation (3) normalized by the total mass of all the particles $M_{T}$ gives

$$
\frac{M\left(d_{i}\right)}{M_{T}} \propto d^{3-D} .
$$

Following (4), the fractal dimension of the particle-size distribution can be determined from the slope of $\log \left[M\left(d_{i}\right) / M_{T}\right]$ versus $\log d$. 


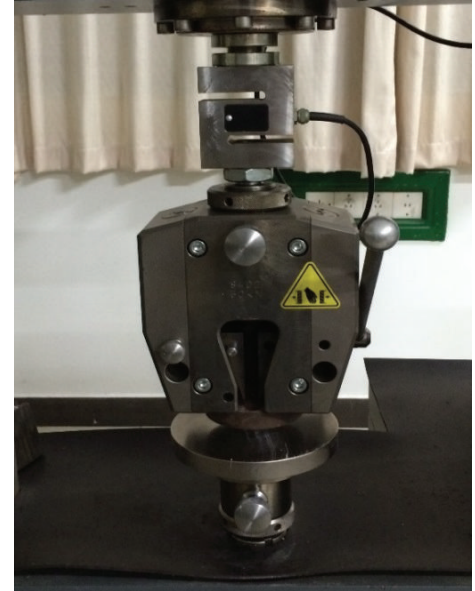

(a)

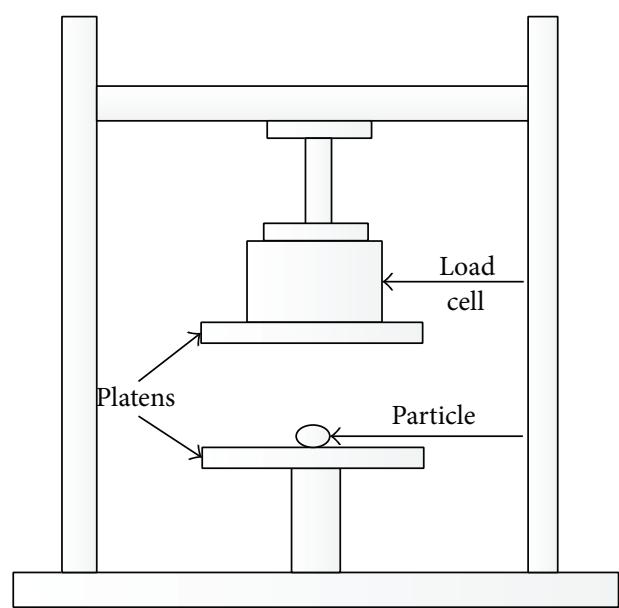

(b)

FIgURE 1: The apparatus of particle crushing test.

\section{Single Particle Crushing Tests}

3.1. Material and Apparatus. The material used for the experiment is marble pebble, an actual rock, the main components of which are $\mathrm{CaCO}_{3}, \mathrm{MgCO}_{3}$, and $\mathrm{SiO}_{2}$, with impurities of $\mathrm{Al}_{2} \mathrm{O}_{3}$ and $\mathrm{Fe}_{2} \mathrm{O}_{3}$. The characteristic diameters of the particles used for the experiment range from $6.0 \mathrm{~mm}$ to $32.0 \mathrm{~mm}$. The shapes of the particles are not perfectly spherical, so it has no way to using "diameter" in the strict sense to describe particle size. Then characteristic diameter is employed to express the size of the particles, which is defined as $d=\left(d_{1} d_{2} d_{3}\right)^{1 / 3}$, where $d_{1}, d_{2}$, and $d_{3}$ are the lengths of each particle measured along three different axes.

Figure 1 contains an apparatus of particle crushing test. The test is carried out by transducer placing the particle between two hardened platens and then bringing the upper platen downwards at a constant velocity in order to crush the particle. The transducer diameter of the removable hardened platens is $150 \mathrm{~mm}$, and the load-measuring capacity of the apparatus is $50 \mathrm{kN}$, with a resolution of $0.01 \mathrm{kN}$. The transducers measuring the displacement and the force are arranged on the instrument, which can collect and record data automatically.

3.2. Methods. The purpose of experiments is to get fractal crushing and mechanical properties of marble particles using single particle crushing tests. The fractal dimension of particle crushing is measured from the particle size distribution of particle fragmentation. A total of 600 single particle crushing tests are carried out on marble particles.

The rock particles are divided into six groups according to their characteristic particle size: $6.0-8.0 \mathrm{~mm}, 8.0-$ $10.0 \mathrm{~mm}, 10.0-13.0 \mathrm{~mm}, 13.0-16.0 \mathrm{~mm}, 16.0-25.0 \mathrm{~mm}$, and $25.0-33.0 \mathrm{~mm}$, with each group containing 100 particles. This will ensure not only the accuracy of the experimental results, but also the controllability of time cost. Before the test, single particle is placed on the centre of the bottom platen for preparation. The test commences with a loading velocity of

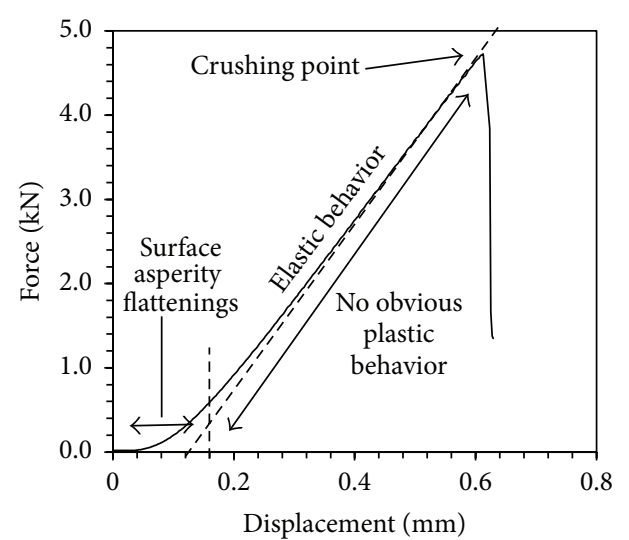

FIGURE 2: Force-displacement relationship before primary crushing.

$1.0 \mathrm{~mm} / \mathrm{min}$ that continues until the particles are crushed, with the crushed particles then being collected for the sieve tests. The instrument is able to automatically capture force and displacement data in real time during tests. Since the loading velocity is $1 \mathrm{~mm} / \mathrm{min}$ and the characteristic particle sizes are in range of $6.0-33.0 \mathrm{~mm}$, the strain rates range from $5.05 \times 10^{-4}$ to $2.78 \times 10^{-3} \mathrm{~s}^{-1}$. The loading velocity has been chosen to guarantee quasistatic loading of the particles.

3.3. Results and Discussion. Figure 2 shows the typical forcedisplacement behaviour of single marble particle in uniaxial compression tests. Prior to diametrical loading, a contact arises between the particle surface and the punch surfaces where negligible deformation and yielding of the surface asperities occur. This phenomenon is the same as that presented by Russell et al. $[10,11]$. On diametrical loading, the particle undergoes elastic contact deformation until reaching the crushing point. The breakages are brittle; no obvious plastic behavior occurs in Figure 2 . 


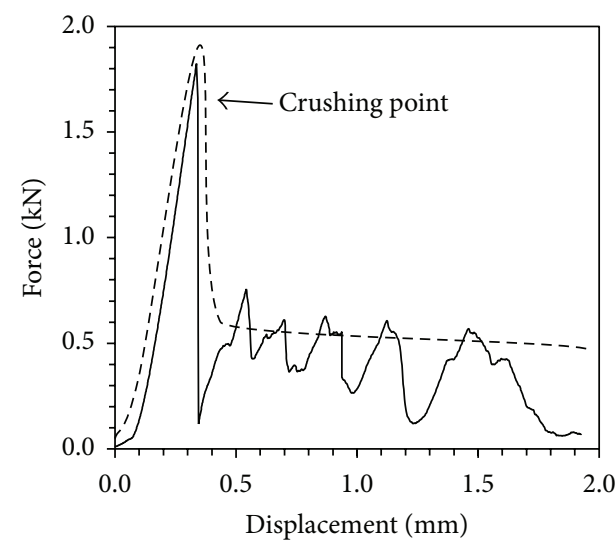

(a) Type I

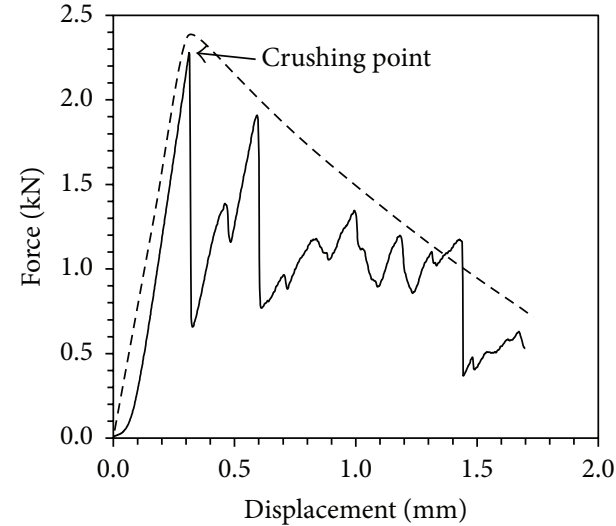

(b) Type II

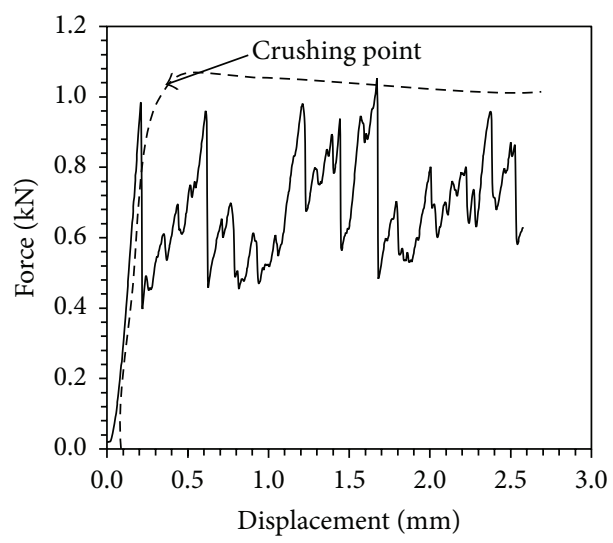

(c) Type III

FIgURE 3: Typical force-displacement relationship.

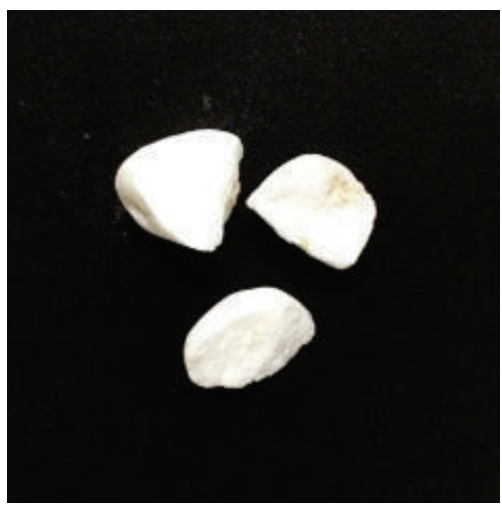

(a) Type I

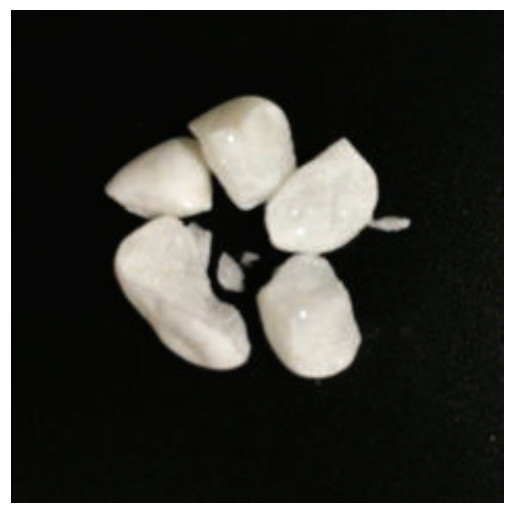

(b) Type II

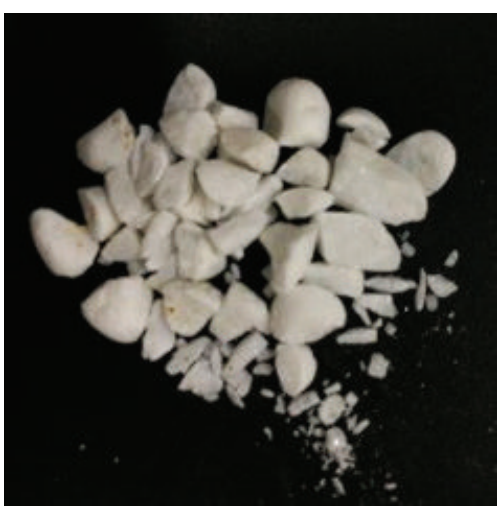

(c) Type III

FIgURE 4: Mother particle and its fragments.

When the force reaches the crushing point as the load increases, the particle will be primarily crushed. After the primary crushing, force and displacement curve is different for different particles. Figure 3 shows three typical and complete processes of force-displacement relationships obtained from the particle crushing tests. Type I compression curve is shown in Figure 3(a). The primary crushing occurs when the stress reaches a maximum of around $1.8 \mathrm{kN}$ at the displacement of $0.35 \mathrm{~mm}$. Then the stress suddenly decreases and after that always remains at low level. This type of particles tested tends to form only two or three fragments after crushing, as shown in Figure 4(a), and hence may be described as incompletely crushed. Type II compression curve is shown in Figure 3(b), and while the force still decreases after the first 


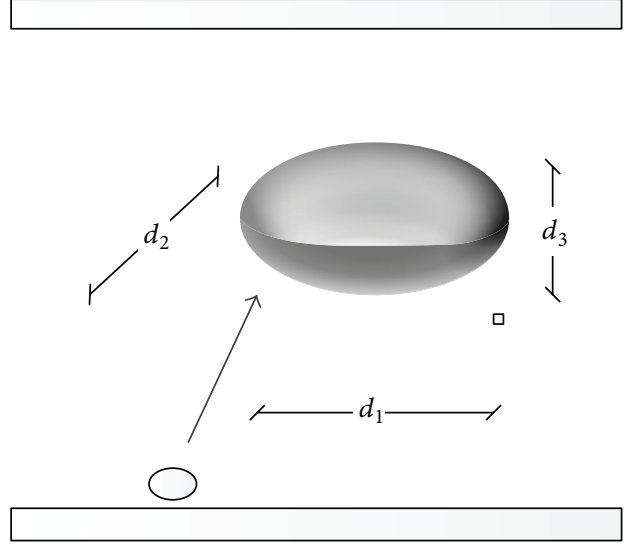

FIGURE 5: Shape parameter of particle.

peak is reached, but compared with type I, this curve is slowly and stepwisely reduced. This type of particle breaks into several small fragments when crushed, as seen in Figure 4(b), which is also an incompletely crushed particle type. In type III compression curve, as shown in Figure 3(c), the forcedisplacement curve may reach a second peak that is even higher than the first peak. The particles seen in Figure 4(c) form into many tiny fragments and hence are completely crushed. It is noted that the different types of particle crushing are a result of the irregular shapes and flaws of particles.

The diameters of each particle in three directions are, in descending order, $d_{1}>d_{2}>d_{3}$, as shown in Figure 5. Also, they are perpendicular to each other. As the data for each particle are 3D, two aspect ratios are calculated, the elongation index (EI) and the flatness index (FI), which are defined as $\mathrm{EI}=d_{2} / d_{1}$ and FI $=d_{3} / d_{1}$. The shape of the particles is "lanker" when EI is smaller, like a rod, while the bottom of the particles is "round" when EI is large. The smaller the FI value, the more "flat" the particles are, and when both values of EI and FI are close to 1.0, the particles are approximately spherical.

The connection between the crushing type and the shape of the particles is shown in Figure 6. The particles in area III of Figure 6 are inerratic in shape and mostly fit type I forcedisplacement compression curve shown in Figure 3(a). The anomalistic particles fall in the shaded region III and mostly fit type III compression curve, as shown in Figure 3(c). The particles in region II are less uniformly distributed and fit into all three compression curves. The peak forces in Figure 3 each represent the partial crushing of a particle. For inerratic particles, the crushing will be strongest at the first peak as the mother particle is broken into two or three pieces, and after that there will be no further significant crushing. This leads them to possessing type I force-displacement compression curve. For flat particles, the panel of the instrument and the mother particle are in sufficient contact for crushing at the initial stage of loading. This leads to many tiny fragments formation after crushing, manifesting in type III compression curve. Type II curve is an intermediate stage of the other two types.

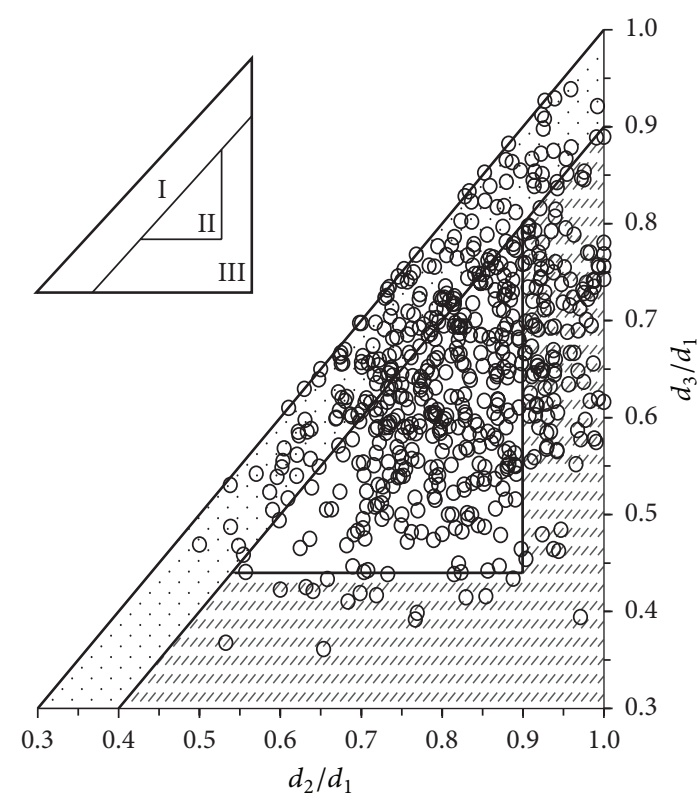

FIGURE 6: Particle shape type.

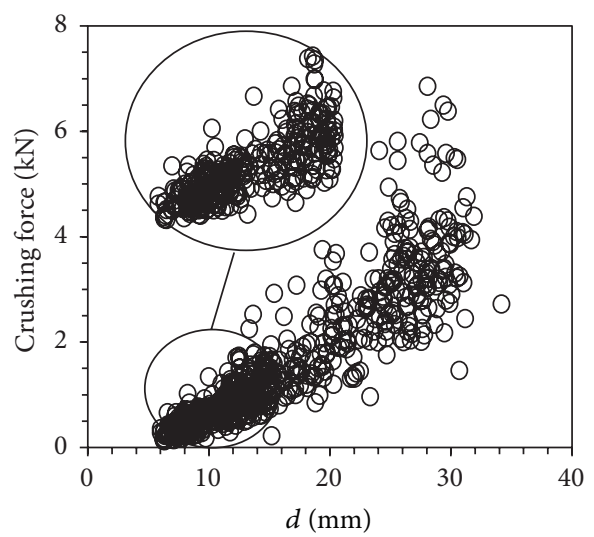

FIGURE 7: Relationship of crushing force and particle size.

The first peak value on the force-displacement compression curve in the single particle crushing tests is defined as the particle crushing force. The relationship between the particle crushing force and the particle size is shown in Figure 7. It can be seen that the particle crushing force grows as particle size increases.

The fractal dimension of the particle crushing may be determined by analysing data from the sieve tests according to (4). The apertures of the sieve tests used are $0.1 \mathrm{~mm}$, $0.5 \mathrm{~mm}, 1.0 \mathrm{~mm}, 2.0 \mathrm{~mm}, 5.0 \mathrm{~mm}, 10.0 \mathrm{~mm}, 14.0 \mathrm{~mm}$, and $20.0 \mathrm{~mm}$. There are some fragments generated by friction and internal defects. These fragments will also be counted in the sieve tests for determining the fractal dimension.

The mass-size distribution of the crushed particles is shown in Figure 8(a). The abscissa represents the particle diameter after crushing, and the ordinate represents the ratio of the cumulative mass under the sieve to the total mass of crushed particles. The minimum diameter of the marble 


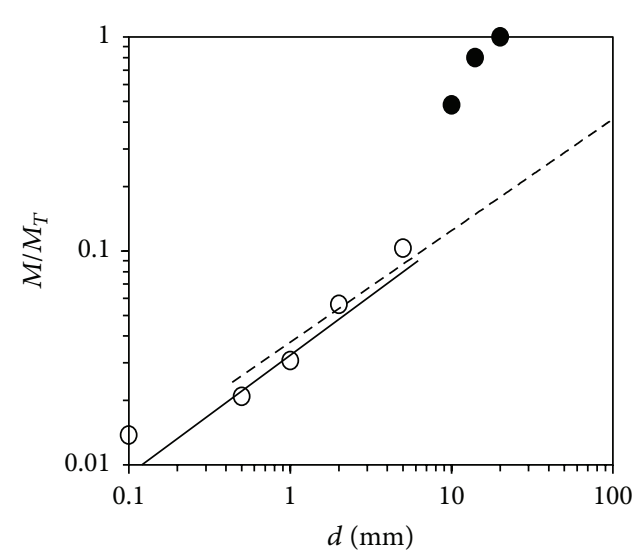

(a) Containing mother particles

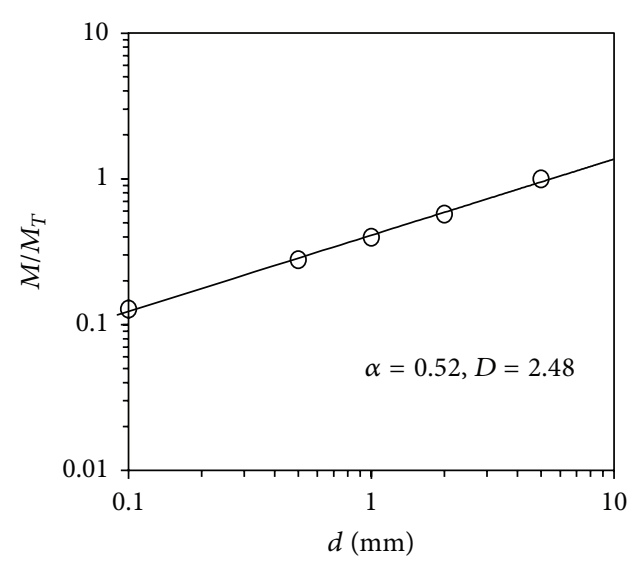

(b) Crushed particles

FIGURE 8: Particle size distribution.

particles is $6.0 \mathrm{~mm}$, and particles with diameter greater than $6.0 \mathrm{~mm}$ are not crushed. It should be noted that the crushed particles with diameter less than $5.0 \mathrm{~mm}(d<$ $5.0 \mathrm{~mm}$ ) demonstrate a linear distribution in $\log$-log that is approximately 0.52 . The mass-size distribution of the particles with diameter greater than $5.0 \mathrm{~mm}$ deviates the loglog coordinates in Figure 8(a). The deviation in Figure 8(a) is due to the fact that particles are not completely crushed. All particles with the diameter less than $5.0 \mathrm{~mm}$ are collected and the mass size distribution of crushed particles is shown in Figure 8(b). It is seen from Figure 8(b) that the masssize distributions of crushed particles in a log-log plot can be described by a linear relationship, whose slope $\alpha$ is approximately 0.52 . According to (4), the fractal dimension of crushed particles $D$ is 2.48 .

It should be noted that the result $D=2.48$ is only suitable for the specific material and the specific failure mode here in this paper. It means that the result might not be applicable to comminution operations where strain rates are very high (likely to be $10^{4}-10^{6} \mathrm{~s}^{-1}$ ). However, this method here is still suitable because the different loading rates will lead to the change of the particle size distribution; then the fractal dimension will also change. In that case, an updated $D$ can be obtained for comminution operations with different strain rates. This also proves that, at higher displacement controlled loading rates, higher compressive loads are required to realize equivalent deformation in comparison to those achieved by loads at lower displacement-controlled loading rates, which has been addressed by Russell et al. [10, 11].

\section{Crushing Strength of Single Particle}

The fractal dimension obtained in the previous section will play an important role in predicting the mechanical properties of the particles. The apparent crushing strength of particles is given by

$$
\sigma_{f}=\frac{F_{f}}{A_{\mathrm{app}}},
$$

where $\sigma_{f}$ is the apparent crushing strength of particles, $F_{f}$ is the ultimate fracture force, and $A_{\text {app }}$ is the apparent area of the particle in the section perpendicular to $F_{f}$. The apparent area of the particle is written as

$$
A_{\mathrm{app}}=k_{1} d^{2}
$$

where $d$ is the particle diameter and $k_{1}$ is a shape factor; when the contact surface between the particle and the platens is square, $k_{1}=1$.

The fractal dimension of the particle size distribution is $D$ in three dimensions; therefore the fractal dimension of the particles size distribution is $D-1$ in two-dimensional crosssectional planes according to the rules of thumb $[16,28]$. The real particle area in any section with size $d$ is written as

$$
A_{\text {real }}=k_{1} d^{D-1}
$$

where $A_{\text {real }}$ is the real section area of the particles. The intrinsic tensile strength $\sigma_{f}^{*}$ of particles is a constant value that does not vary with particle size, which is written as

$$
\sigma_{f}^{*}=\frac{F_{f}}{A_{\text {real }}} .
$$

Combining (5), (6), (7), and (8),

$$
\begin{aligned}
\sigma_{f} & =\sigma_{f}^{*} d^{-\beta}, \\
\beta & =3-D, \\
\sigma_{f}^{*} & =\frac{\sum_{i=2}^{n} \sigma_{f i} d_{i}^{3-D}}{n} .
\end{aligned}
$$

The particle diameter $d$ is difficult to measure, so it is replaced by the characteristic diameter in this section. Figure 9 shows the relationship between crushing strength and characteristic diameter of marble particle in a log-log plot. The solid line in Figure 9 is the prediction of (9). The fractal dimension of crushing particles is 2.48 obtained from the mass size 


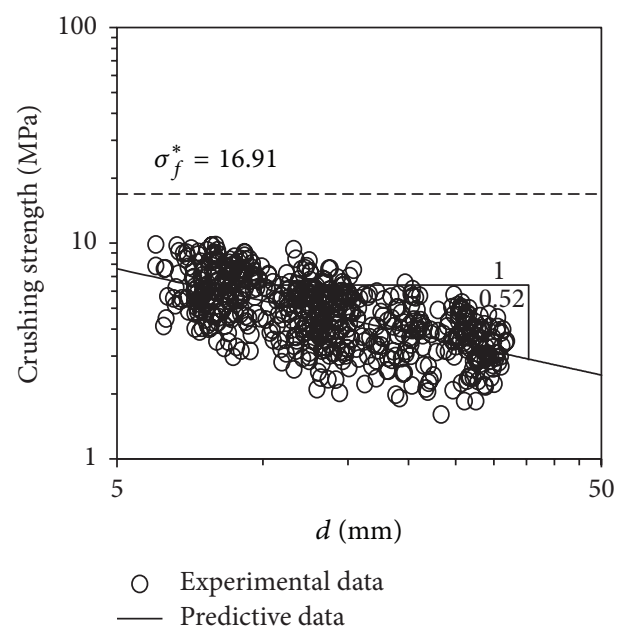

FIGURE 9: Comparisons of predictions with experimental data of crushing strength.

distribution in Figure 8. According to (10), the value of $\beta$ is 0.52 . It is seen from Figure 9 that the prediction of (9) matches well with the experimental data. It is seen however from Figure 9 that the discrete value of the failure strength is quite high even when the size of the particles is the same, which is mainly due to the influence of the irregularity of the particles. Since even particles are of the same size, their shape may not be exactly the same. Thus, their contact surfaces may differ, and their crushing strength is not necessarily the same.

The probability of survival at a given crushing stress $\sigma_{c}$ is defined as

$$
\begin{aligned}
P_{s}= & \frac{\text { Amount of Crushed Particles at } \sigma>\sigma_{c}}{\text { Total Amount of Particles }} \\
& \times 100 \% .
\end{aligned}
$$

Figure 10 shows the curves representing the probabilities of survival under a given characteristic stress $\sigma_{c}$. Curves are presented for the particle size ranges of $6.0-8.0 \mathrm{~mm}, 8.0-$ $10.0 \mathrm{~mm}, 10.0-13.0 \mathrm{~mm}, 13.0-16.0 \mathrm{~mm}, 16.0-25.0 \mathrm{~mm}$, and $25.0-33.0 \mathrm{~mm}$, respectively.

The survival probability $P_{s}(\sigma, n)$ of granular material can be calculated from [25]

$$
P_{s}(\sigma, n)=\exp \left[-n\left(\frac{\sigma}{\sigma_{0}}\right)^{m}\right] \text {, }
$$

where $P_{s}(\sigma, n)$ is the probability of survival of any particle subjected to a stress $\sigma, \sigma_{0}$ is the characteristic stress at which $37 \%$ of particles survive, $m$ is the Weibull modulus, and $n=$ $\left(d / d_{0}\right)^{3}$. In the fractal model, $n=\left(d / d_{0}\right)^{D}$, so (13) can be expressed as

$$
P_{s}(d, n)=\exp \left[-\left(\frac{d}{d_{0}}\right)^{D}\left(\frac{\sigma}{\sigma_{0}}\right)^{m}\right]
$$

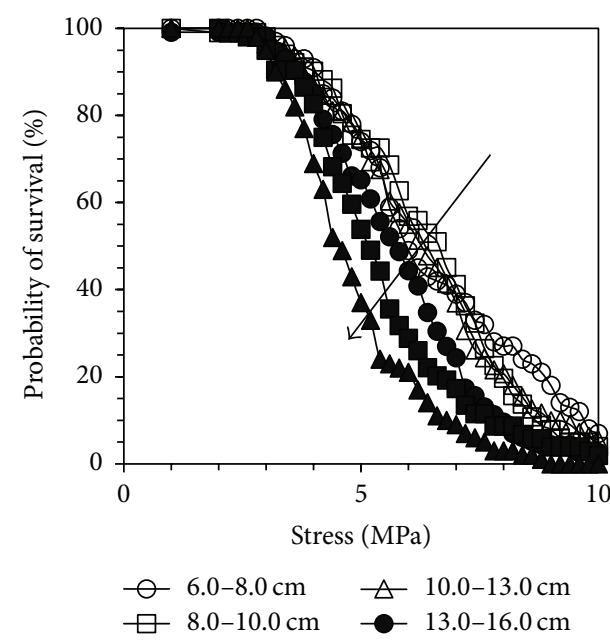

FIgURE 10: Relationship of probability of survival and crushing strength.

If the tensile strength is defined as tensile stress at a standard probability of survival for various batches of particles with diameter $d$, it may be expressed as

$$
\left(\frac{d}{d_{0}}\right)^{D}\left(\frac{\sigma_{f}}{\sigma_{f 0}}\right)^{m}=\text { const. }
$$

Combining with (9) gives

$$
m=\frac{D}{3-D}
$$

It can be seen that $\ln \left[\ln \left(1 / P_{s}\right)\right]$ increases linearly as $\ln \left(\sigma / \sigma_{0}\right)$ increases, as shown in Figure 11, and the slope is $m$. Taking the experiment result $D=2.48$ in (16), it can be calculated that $m=4.77$. The predictions of (14) based on the use of a fractal dimension of 2.48 are also plotted in Figure 11. The predictions of (14) match satisfactorily with the experimental data.

\section{Crushing Energy of Single Particle}

The experimental system is set up to detect the force at which particle crushing occurs. The crushing energy is the integration of the crushing force with respect to displacement of the particle as shown in

$$
E_{f}=\int_{0}^{\delta_{f}} F \mathrm{~d} \delta .
$$

In Figure 3, a linear relationship may be observed between the crushing force and displacement before the crushing point; hence the crushing energy is

$$
E_{f}=\frac{1}{2} F_{f} \delta_{f} .
$$

Figure 12 shows the relationship between particle displacement $\delta_{f}$ and the characteristic diameter $d$. The experiment 

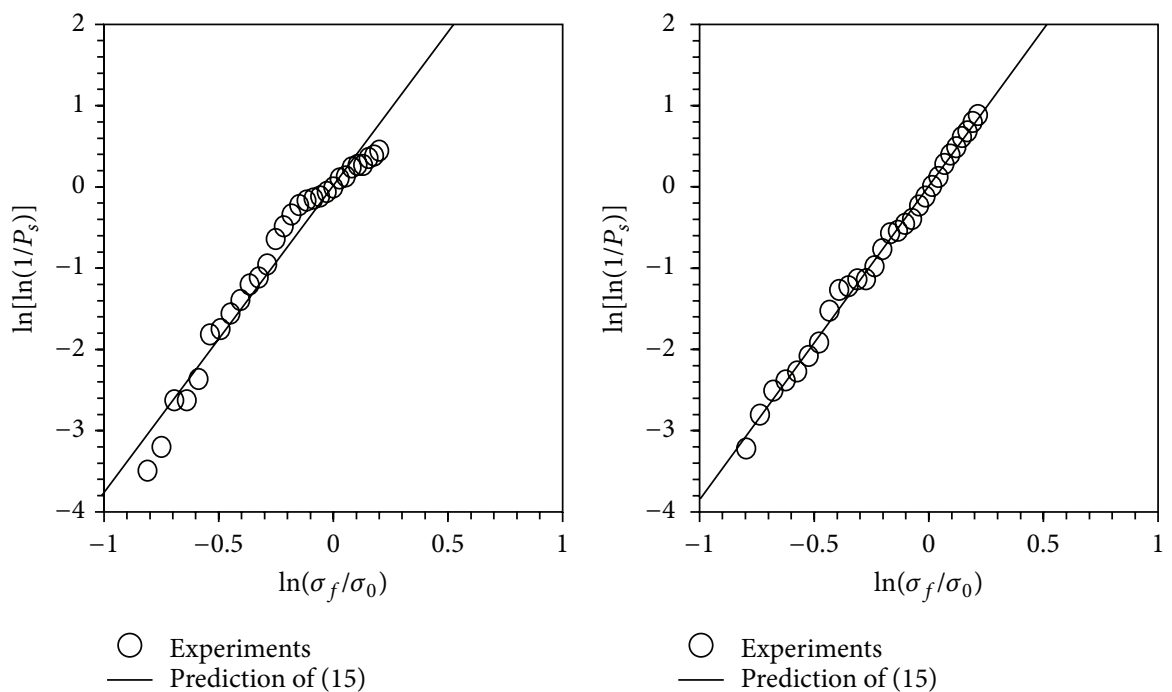

Prediction of (15)

(a) $6.0-8.0 \mathrm{~cm}$

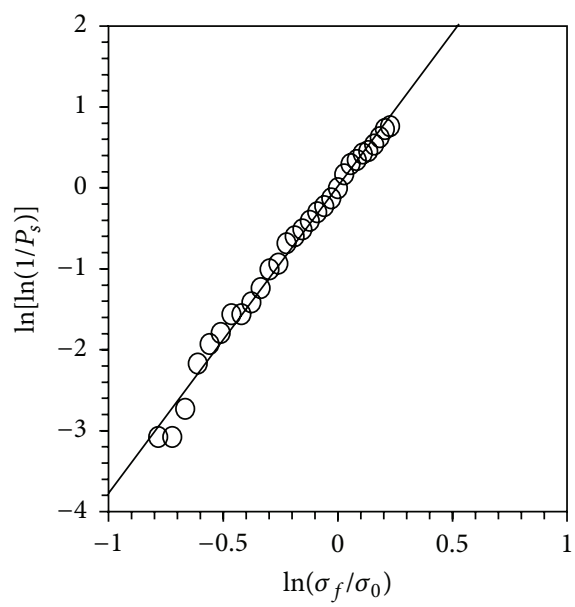

(b) $8.0-10.0 \mathrm{~cm}$

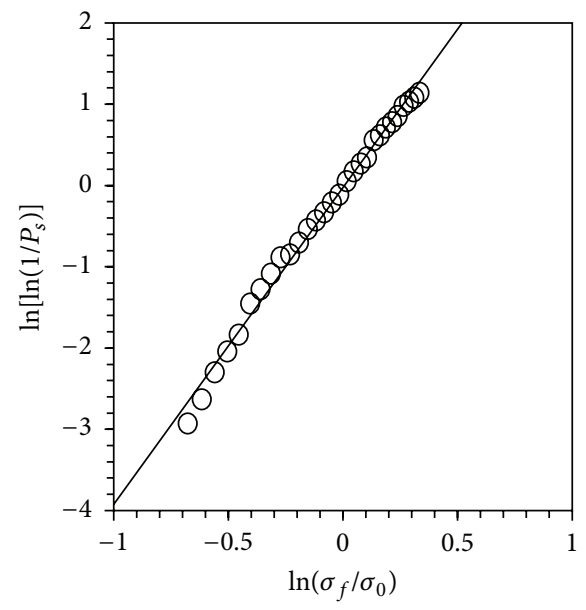

Experiments

Prediction of (15)

(c) $10.0-13.0 \mathrm{~cm}$

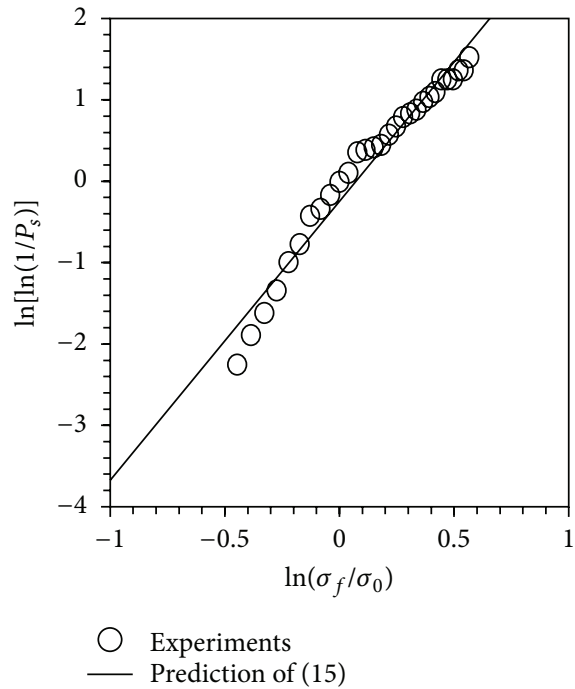

(e) $16.0-25.0 \mathrm{~cm}$

$\bigcirc$ Experiments

Prediction of (15)

(d) $13.0-16.0 \mathrm{~cm}$



(f) $25.0-33.0 \mathrm{~cm}$

FIGURE 11: Comparison between predictions of (15) and experiments. 


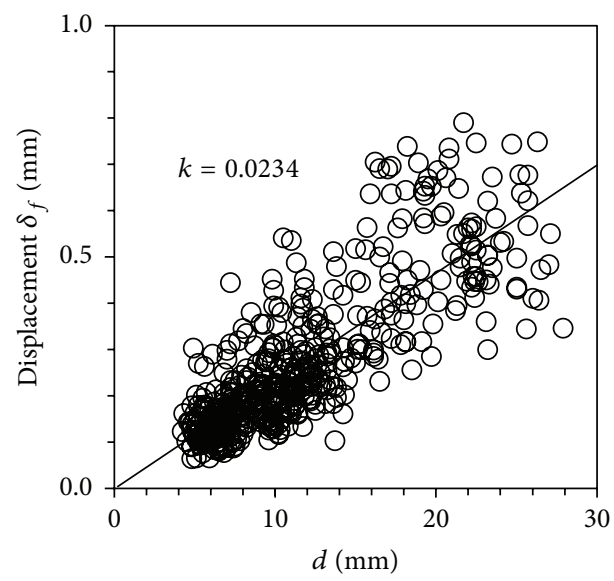

Figure 12: Relationship between $\delta_{f}$ and $d$.

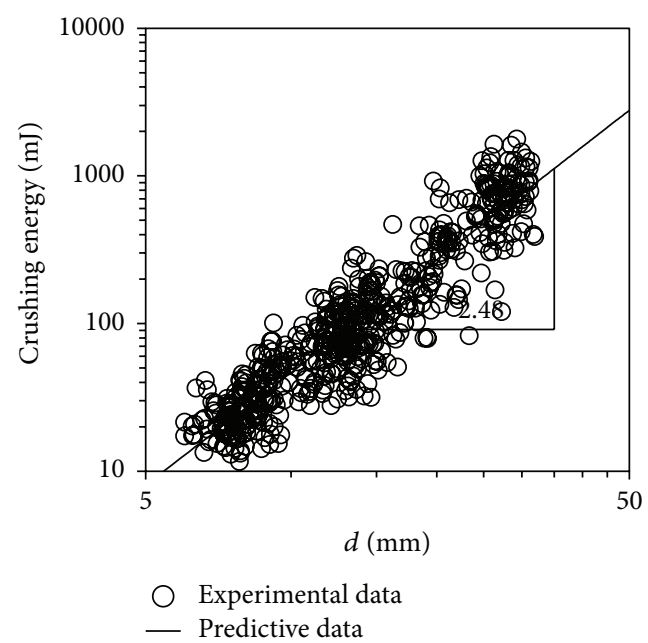

FIGURE 13: Comparison of predictions with experimental data of crushing energy.

reveals that the displacement $\delta_{f}$ and diameter $d$ are directly proportional:

$$
\delta_{f} \propto d_{3} \propto d=k_{2} d,
$$

where $k_{2}$ is the proportionality coefficient between $\delta_{f}$ and $d$.

Substitute (7), (8), and (19) into (18),

$$
E_{f}=k_{3} d^{D},
$$

where $k_{3}$ is a constant and $k_{3}=0.5 k_{1} k_{2} \sigma_{f}^{*}$. So it is a linear relationship between the crushing energy and the diameter of the particles in log-log coordinates, and the slope should equal the fractal dimension $D$.

Figure 13 contains all the experimental data of crushing energy. The relationship between crushing energy and characteristic diameter is shown in a log-log plot. The relationship between crushing energy and characteristic diameter is an approximate line, with the slope of 2.48 , which is equal to the fractal dimension $D=2.48$ of crushing particles.

\section{Conclusions}

(1) Uniaxial compression tests on single particle reveal that the force-displacement curves of marble particles are divided into three types depending on the particle shape.

(2) Particle crushing occurs in uniaxial compression tests on single particle. The particle size distribution of crushed particles obeys fractal model with the fractal dimension of 2.48 for marble particles. The fractal dimension of crushed particles should be calculated from the particle size distribution of completely crushed particles.

(3) The relationship between the crushing strength and particle size is presented in the fractal model for the particle crushing. The proposed relationship between the crushing strength and particle size is verified by experiments of marble particles.

(4) Based on the fractal model for the particle crushing, the probability of survival and crushing energy of marble particles is also derived and verified by experiments.

(5) In this paper, the marble particles are selected to conduct the particle crushing tests and to derive the fractal model for crushing. However, the fractal model is not limited to marble particles and can be conveniently applied to the other particles. In addition, a simple and goodness-of-fit method is proposed to predict the crushing strength, the crushing energy, and the probability of survival of particles based on the fractal fragmentation of marble particles.

\section{Conflict of Interests}

The authors declare that there is no conflict of interests regarding the publication of this paper.

\section{Acknowledgment}

The National Nature Science Foundation of China under Grants nos. 41272318 and 41472251 is acknowledged for its financial support.

\section{References}

[1] J. H. Wang, X. L. Zhou, and J. F. Lu, "Dynamic response of pile groups embedded in a poroelastic medium," Soil Dynamics and Earthquake Engineering, vol. 23, no. 3, pp. 235-242, 2003.

[2] J.-J. Chen, L. Y. Zhang, J.-F. Zhang, Y.-F. Zhu, and J.-H. Wang, "Field tests, modification, and application of deep soil mixing method in soft clay," Journal of Geotechnical and Geoenvironmental Engineering, vol. 139, no. 1, pp. 24-34, 2013.

[3] I. Einav, "Breakage mechanics-part I: theory," Journal of the Mechanics and Physics of Solids, vol. 55, no. 6, pp. 1274-1297, 2007.

[4] M. Takei, O. Kusakabe, and T. Hayashi, "Time-dependent behavior of crushable materials in one-dimensional compression tests," Soils and Foundations, vol. 41, no. 1, pp. 97-121, 2001. 
[5] Y. Nakata, A. F. L. Hyde, M. Hyodo, and H. Murata, "A probabilistic approach to sand particle crushing in the triaxial test," Geotechnique, vol. 49, no. 5, pp. 567-583, 1999.

[6] K. Matsui, T. Sekiguchi, and H. Ikahata, "Fundamental studies on slow compression packing and crushing of powdery layers," Kagaku Kogaku, vol. 35, no. 7, pp. 788-793, 1971.

[7] Y. Rozenblat, D. Portnikov, A. Levy, H. Kalman, S. Aman, and J. Tomas, "Strength distribution of particles under compression," Powder Technology, vol. 208, no. 1, pp. 215-224, 2011.

[8] R. J. Marsal, Research on Granular Materials: (Rockfills and SoilGravel Mixtures), Instituto de Ingeniería, Universidad Nacional Autónoma de México, 1977.

[9] M. Oda and H. Kazama, "Microstructure of shear bands and its relation to the mechanisms of dilatancy and failure of dense granular soils," Geotechnique, vol. 48, no. 4, pp. 465-481, 1998.

[10] A. Russell, P. Müller, H. Shi, and J. Tomas, "Influences of loading rate and preloading on the mechanical properties of dry elastoplastic granules under compression," AIChE Journal, vol. 60, no. 12, pp. 4037-4050, 2014.

[11] A. Russell, P. Müller, and J. Tomas, "Quasi-static diametrical compression of characteristic elastic-plastic granules: energetic aspects at contact," Chemical Engineering Science, vol. 114, pp. 70-84, 2014.

[12] A. Russell, J. Schmelzer, P. Müller, M. Krüger, and J. Tomas, "Mechanical properties and failure probability of compact agglomerates," Powder Technology, vol. 286, pp. 546-556, 2015.

[13] K. Mader-Arndt, Z. Kutelova, R. Fuchs et al., "Single particle contact versus particle packing behavior: model based analysis of chemically modified glass particles," Granular Matter, vol. 16, no. 3, pp. 359-375, 2014.

[14] L. Ribas, G. C. Cordeiro, R. D. Toledo Filho, and L. M. Tavares, "Measuring the strength of irregularly-shaped fine particles in a microcompression tester," Minerals Engineering, vol. 65, pp. 149-155, 2014.

[15] D. Portnikov, H. Kalman, S. Aman, and J. Tomas, "Investigating the testing procedure limits for measuring particle strength distribution," Powder Technology, vol. 237, pp. 489-496, 2013.

[16] B. B. Mandelbrot, The Fractal Geometry of Nature, W. H. Freeman, San Francisco, Calif, USA, 1982.

[17] A. Carpinteri and N. Pugno, "A multifractal comminution approach for drilling scaling laws," Powder Technology, vol. 131, no. 1, pp. 93-98, 2003.

[18] Y. F. Xu, "Explanation of scaling phenomenon based on fractal fragmentation," Mechanics Research Communications, vol. 32, no. 2, pp. 209-220, 2005.

[19] T. Allen, Powder Sampling and Particle Size Determination, Elsevier Science, 2003.

[20] P. Lu, I. F. Jefferson, M. S. Rosenbaum, and I. J. Smalley, "Fractal characteristics of loess formation: evidence from laboratory experiments," Engineering Geology, vol. 69, no. 3-4, pp. 287-293, 2003.

[21] H. A. Ahmed and J. Drzymała, "Two-dimensional fractal linearization of distribution curves," Physico-Chemical Problems of Mineral Processing, vol. 39, pp. 129-139, 2005.

[22] F. Casini, G. M. B. Viggiani, and S. M. Springman, "Breakage of an artificial crushable material under loading," Granular Matter, vol. 15, no. 5, pp. 661-673, 2013.

[23] G. R. McDowell, M. D. Bolton, and D. Robertson, "Fractal crushing of granular materials," Journal of the Mechanics and Physics of Solids, vol. 44, no. 12, pp. 2079-2102, 1996.
[24] Y. F. Xu, J. Xu, and J. Wang, "Fractal model for size effect on ice failure strength," Cold Regions Science and Technology, vol. 40, no. 1-2, pp. 135-144, 2004.

[25] W. Weibull, "A statistical distribution function of wide applicability," Journal of Applied Mechanics, vol. 18, pp. 293-297, 1951.

[26] B. D. Zhao, J. F. Wang, M. R. Coop, G. Viggiani, and M. Jiang, "An investigation of single sand particle fracture using $\mathrm{x}$-ray micro-tomography," Géotechnique, vol. 65, no. 8, pp. 625-641, 2015.

[27] Y. F. Xu and P. Dong, "Fractal approach to hydraulic properties in unsaturated porous media," Chaos Solitons \& Fractals, vol. 19, no. 2, pp. 327-337, 2004.

[28] Y. F. Xu and D. A. Sun, "Determination of expansive soil strength using a fractal model," Fractals, vol. 9, no. 1, pp. 51-60, 2001. 

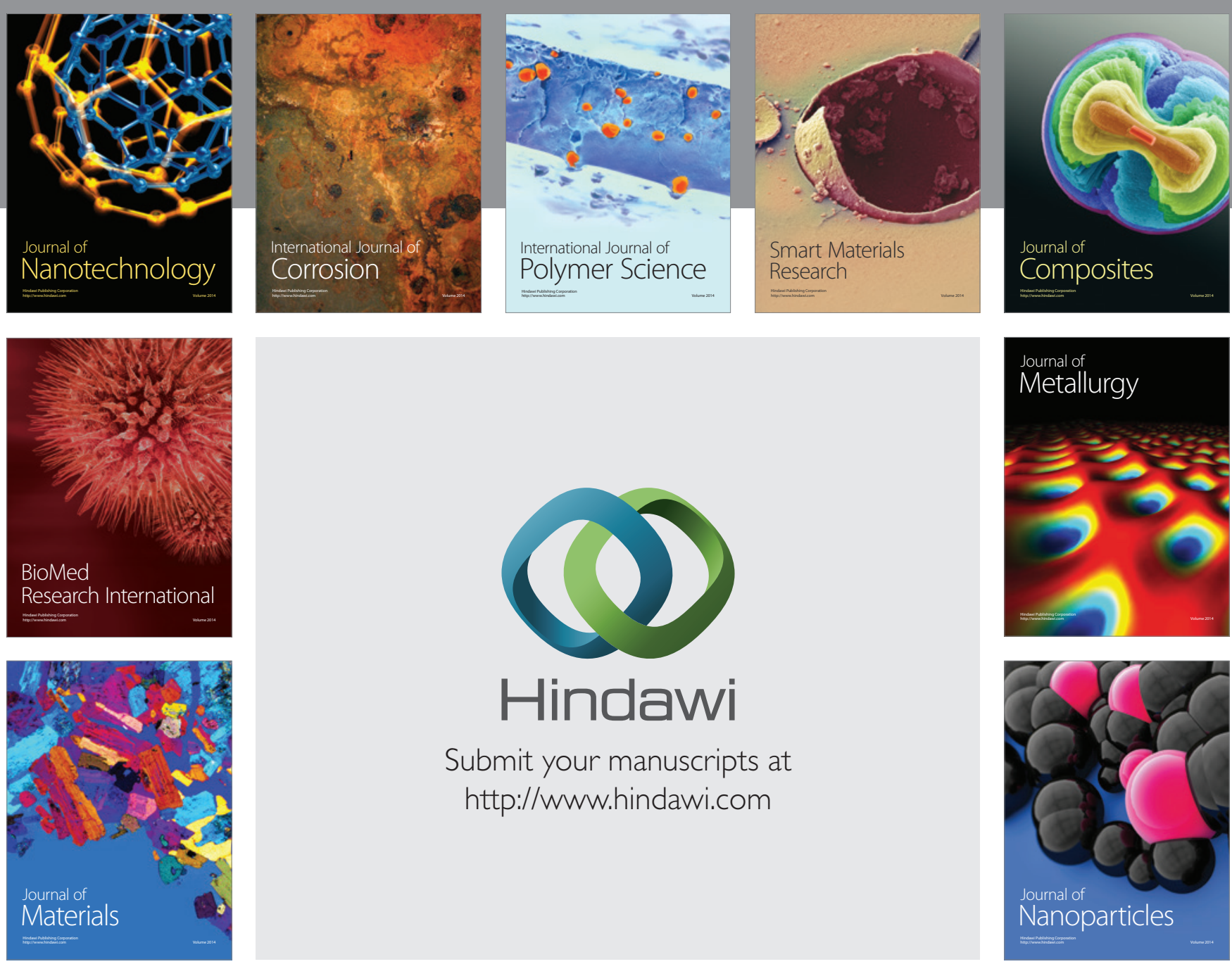

Submit your manuscripts at http://www.hindawi.com
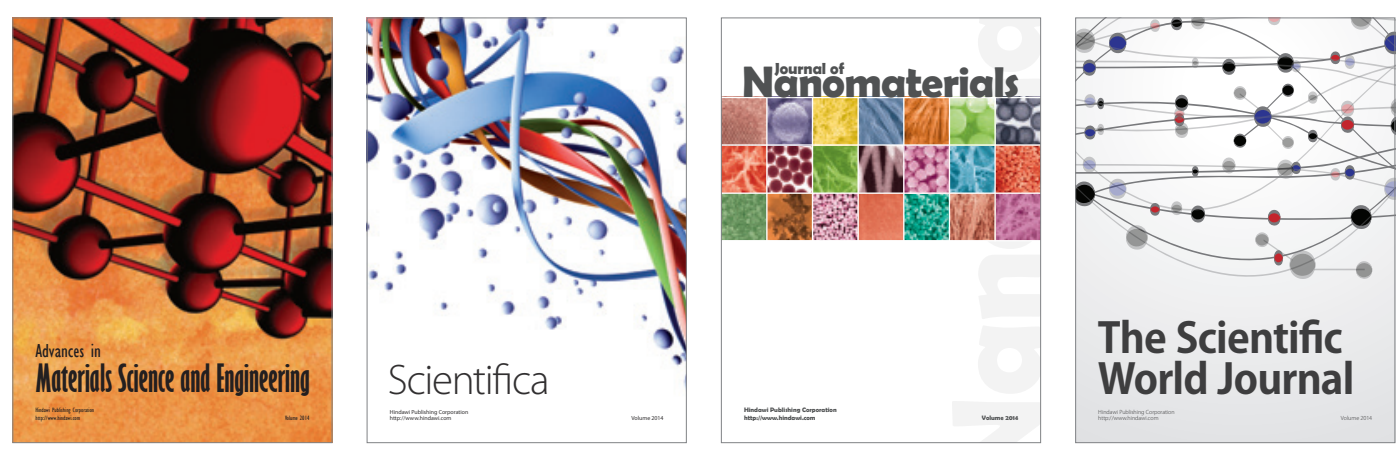

\section{The Scientific World Journal}
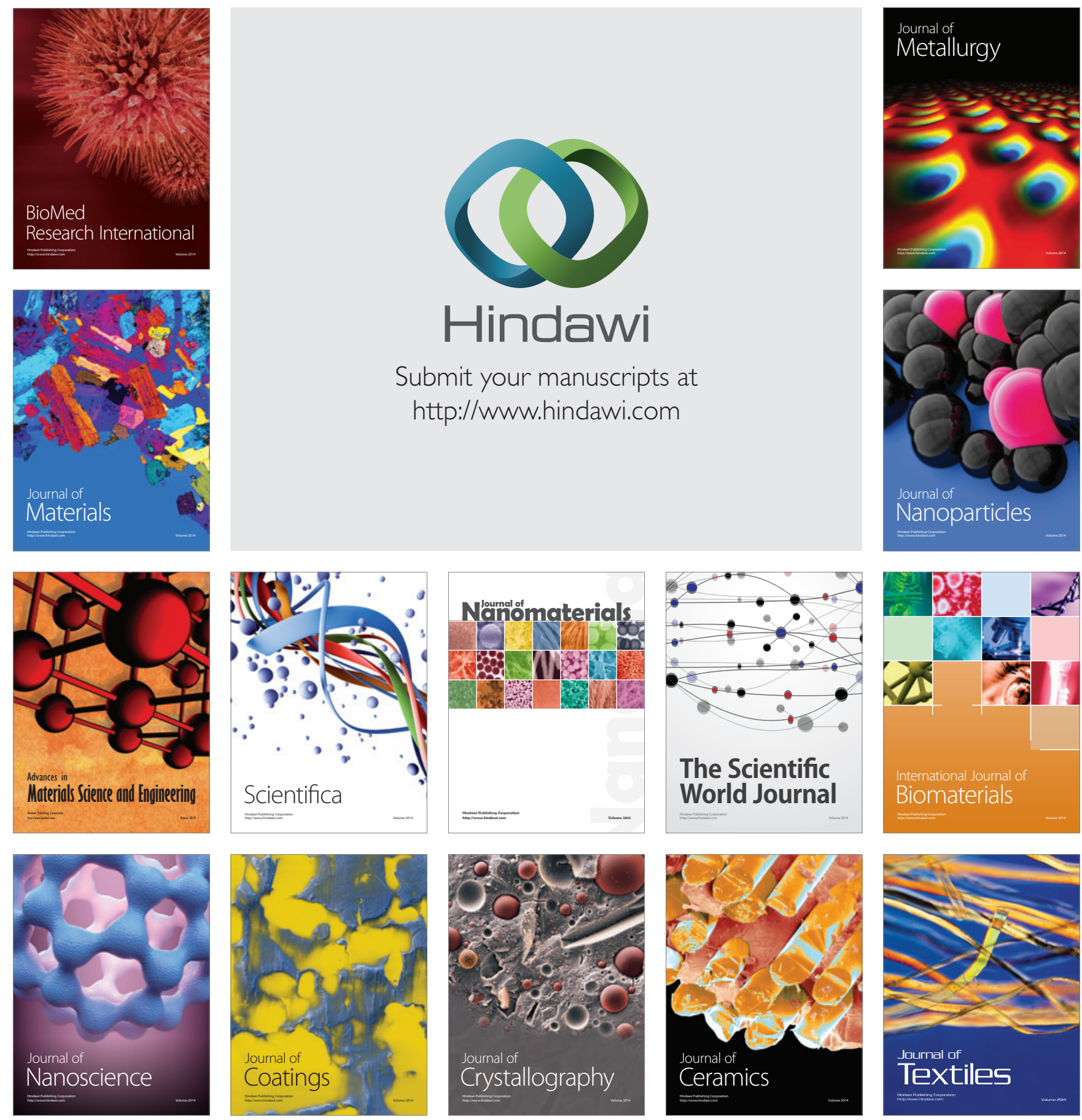